\title{
Matrix viscoelasticity controls spatio-temporal tissue organization
}

Alberto Elosegui-Artola ${ }^{1,2,3, \#}$, Anupam Gupta1,\#, Alexander J. Najibi ${ }^{1,2}$, Bo Ri Seo ${ }^{1,2}$, Ryan Garry ${ }^{1}$, Max Darnell ${ }^{1,2}$, Wei Gu${ }^{4}$, Qiao Zhou ${ }^{4}$, David A. Weitz ${ }^{1,5}$, L. Mahadevan ${ }^{1,5,6^{*}}$, David J. Mooney ${ }^{1,2^{*}}$

1.- Harvard John A. Paulson School of Engineering and Applied Sciences, Harvard University, Cambridge, MA, USA

2.- Wyss Institute for Biologically Inspired Engineering, Cambridge, MA, USA

3.- Institute for Bioengineering of Catalonia, Barcelona, Spain.

4.- Weill Cornell Medicine, Cornell University, NY, USA

5.- Department of Physics, Harvard University, Cambridge, United States, MA, USA

6.- Department of Organismic and Evolutionary Biology, Harvard University, Cambridge, MA, USA

\# Equal contributions

* Corresponding authors 
1 The spatio-temporal patterning of multicellular tissues is driven by the collective dynamics

2 of cell proliferation and active movement. These processes are mediated by the

3 extracellular matrix environment via a combination of biomolecular and physical cues.

4 Here we show that the passive viscoelastic properties of the matrix that encapsulate a

5 proliferating ball of cells (e.g. a developing organoid) play a critical role in guiding tissue

6 organization in space and time. By varying the viscoelasticity of well-defined model

7 matrices, we show how a spheroidal tissue of breast epithelial cells breaks symmetry and

8 forms finger-like protrusions that invade the matrix. A computational model allows us to

9 recapitulate these observations and leads to a phase diagram that demarcates the regions

10 of morphological stability and instability as a function of matrix viscoelasticity, tissue

11 viscosity, cell motility and cell division rate. Experiments that use biomolecular

12 manipulations to independently vary these parameters confirm our predictions. To further

13 test our theory, we also study the self-organization of an in-vitro intestinal organoid and

14 show that the morphological changes of this system also fits within our paradigm.

15 Altogether, our studies demonstrate the role of stress relaxation mechanisms in

16 determining the dynamics of tissue growth and the symmetry breaking instabilities

17 associated with branching, a fundamental process in morphogenesis and oncogenesis,

18 and suggest ways of controlling tissue form using the extracellular matrix.

20 Introduction The patterning of tissues in space and time is relevant for many fundamental

21 biological processes (e.g., embryonic development, organogenesis, oncogenesis) ${ }^{1-5}$, and is

22 driven by cell number, size, shape and position changes and leads to symmetry breaking

23 instabilities such as buckling, folding, tearing, budding or branching ${ }^{6-9}$. At a molecular level, the

24 spatio-temporal organization of tissues is regulated by intrinsic gene expression ${ }^{10}$, and a variety

25 of environmental chemical and mechanical cues ${ }^{11}$. While the importance of chemical morphogen 
26 gradients in development has long been appreciated ${ }^{12,13}$, it is increasingly clear that diverse

27 mechanical cues ${ }^{14-19}$ in the tissue and the surrounding 3D extracellular matrix (ECM) also regulate

28 tissue organization and morphogenesis. In particular, the dynamic interaction between cell

29 behavior and the matrix, with its time-varying mechanical properties is increasingly thought to be

30 an important player in morphogenesis ${ }^{20-22}$. Thus, tissue organization is expected to be impacted

31 by the viscoelastic properties of the matrix ${ }^{23}$ whose behaviors vary from an elastic solid-like

32 response to a liquid-like viscous response, with stress relaxation time scales that range from a

33 second to a few hundred seconds ${ }^{20,24}$. Here we report an experimental and computational study

34 of the role of the viscoelasticity of well-defined model matrices in regulating tissue organization in

35 two commonly used in-vitro models of development and pathology, breast epithelial growth ${ }^{25}$ and

36 intestinal organoid development ${ }^{1}$. These studies demonstrate the role of stress relaxation in

37 determining the dynamics of tissue growth and the symmetry breaking instabilities associated

38 with branching, a fundamental process in morphogenesis and oncogenesis.

Results

\section{Matrix viscoelasticity regulates breast epithelial tissue organization}

41 We first studied the importance of matrix viscoelasticity in the organization and growth of mammary tissues from spheroids of MCF10A non-malignant breast epithelial cells. Hydrogels formed from the natural polysaccharide alginate were chosen as the model matrix system for

44 these studies, as mammalian cells do not express enzymes to degrade these polymers, allowing 45 effects related to matrix degradation to be eliminated ${ }^{26}$. The relative viscoelastic properties of 46 these gels can be readily altered independently of the stiffness, pore size and adhesive ligands ${ }^{24}$.

47 This was achieved here by changing the molecular weight of alginate and the calcium crosslinker 48 density in concert (Fig. 1a) to create gel matrices of constant elastic moduli (G' 5000Pa) (Fig.1b), 49 but varying stress relaxation times $\left(\tau_{\mathrm{m}} \epsilon[30-350 s]\right)$ to achieve matrices that are more elastic 
$50\left(\tau_{\mathrm{m}} \sim 350 \mathrm{~s}\right)$, or more viscoelastic $\left(\tau_{\mathrm{m}} \sim 30 \mathrm{~s}\right)$ (Fig. 1c). As alginate does not present intrinsic integrin

51 adhesion ligands, Arg-Gly-Asp (RGD) containing peptides were conjugated to the polymer

52 backbone to provide a constant level of cell binding sites in all gels ${ }^{27}$. MCF10A breast epithelial

53 cells, widely used to study mammary development and oncogenesis ${ }^{25}$, were formed into

54 spheroids composed of $\sim 2000$ cells and encapsulated in elastic and viscoelastic hydrogels.

55 Over time, tissues in elastic matrices grew slowly and were morphologically stable; they increased

56 in size while maintaining their spherical symmetry. However, tissues in viscoelastic matrices grew

57 much faster. As they increased in size, they exhibited a morphological instability of the nominally

58 smooth tissue-matrix interface; eventually the tissues broke spherical symmetry, formed

59 branches, and invaded the matrix leading to a significant increase in the surface area and a

60 decrease in circularity (Fig.1d-f, Extended Data Fig.1a and Video S1). This is similar to the

61 behavior seen in many biological processes that demonstrate symmetry breaking accompanied

62 by epithelial to mesenchymal transitions $(E M T)^{28}$. In agreement with that precedent, cells in

63 viscoelastic matrices demonstrated an EMT, as vimentin was expressed in branches (Fig. $1 \mathrm{~g})$ and

64 cytokeratin 14 expression was low in cells in spheroids in viscoelastic matrices (Fig.1h,i). To

65 determine whether viscoelasticity enhanced tissue growth in-vivo, MDA-MB-231 malignant breast

66 epithelial cells encapsulated either in viscoelastic or elastic matrices were injected in NOD-SCID

67 mice. Tissues grew significantly more rapidly in viscoelastic rather than in elastic matrices (Fig.1j

68 and Extended Data Fig.2). As all observed differences in-vitro and in-vivo resulted from a change

69 in the mechanical properties of the matrix, our studies next focused on two major

70 mechanosensitive hubs in cells, focal adhesion kinase (FAK) and the mechanosensitive

71 transcriptional regulator Yes-Associated protein (YAP) ${ }^{29}$, both with established roles in MCF10A

$72 \mathrm{EMT}^{2,30,31}$. Viscoelastic, but not elastic matrices promoted the expression of phosphorylated pFAK

73 adhesions (Fig.1k), while YAP remained in the cytoplasm in cells in elastic matrices, but

74 translocated to the nucleus in cells in branches in viscoelastic matrices (Fig.1I,m). When FAK was 
75 inhibited (Extended Data Fig.1b,c), breast epithelium was morphologically stable, confirming the

76 importance of mechanotransduction.

77 Our experiments show that more elastic matrices $\left(\tau_{\mathrm{m}} \sim 350 \mathrm{~s}\right)$, resist tissue invasion, whereas

78 viscoelastic matrices $\left(\tau_{\mathrm{m}} \sim 30 \mathrm{~s}\right)$, are easily invaded by the motile and proliferating cells. Similarly,

79 our observations show that tissues which are highly proliferative lead to an increase in cell influx

80 and likely generate a mechanical pressure that drives the morphological instability of the tissue-

81 matrix interface. These observations of fingering morphologies in active biological systems have

82 physical analogs that have been studied for decades in simple and complex fluids ${ }^{32,33}$. In physical

83 systems, morphological instabilities emerge when driven by pressure gradients (of the right sign)

84 at an interface between contrasting either elastic or viscous properties. More recently, these

85 physical instabilities have been revisited in active matter systems ${ }^{34-36}$. Our experimental

86 observations suggest that the combination of biological activity due to cell migration and/or

87 proliferative pressure at the tissue-matrix interface may lead to a similar symmetry breaking

88 instability exemplified by fingering or branching.

89 Computational model coupling cell motility, proliferative dynamics and matrix viscoelasticity recapitulates tissue organization

91 To understand how the conditions for tissue morphological instability emerge, we consider a 92 minimal theoretical model of the system (Fig.2a and Extended Data Fig.3) starting from a two-

93 phase system of active proliferating cells growing inside a confining passive viscoelastic matrix.

94 We model the individual cells in the tissue as overdamped soft elastic spheres of size $a$ in a liquid

95 of effective viscosity $\mu_{\mathrm{t}}$, which move under the influence of three forces: (i) the interaction

96 between cells, with (a) a short-range repulsion to prevent overlap and (b) mid-range (two cell-

97 length) attraction with the depth in the attractive well $\epsilon$ (see SI for details) which together lead to

98 an active proliferative pressure driven by cell-division, (ii) the repulsion between the cell and the 
surrounding viscoelastic matrix (modeled as a set of similar spheres of size $a$ in a liquid of effective viscosity $\mu_{\mathrm{m}}$ interacting with each other via (a) an attractive potential -equivalent to storage modulus $G^{\prime}$ - and (b) a short-range repulsion to prevent overlap), and (iii) the activity of cells that are assumed to move randomly relative to each other in the bulk, characterized by a motility parameter $M$ (or an effective temperature $)^{37,38}$. Additionally, in the model, the cells at the interface are assumed to have the ability to apply forces on the surrounding matrix ${ }^{39,40}$. The system evolves as cells proliferate and/or migrate actively and the matrix responds passively to the accompanying forces. In particular, the bonds between the spheres in the matrix as well as those between the cells and the matrix can break when strained beyond a prescribed threshold, allowing new bonds to form; this is most likely to happen at the interface between the tissue and the matrix, and allows the boundary between the two phases to evolve dynamically.

111 The parameters in the model allow us to define three dimensionless variables to characterize the 112 scaled matrix fluidity, the passive mechanical relaxation time of the matrix and the relative

113 proliferative capacity of the tissue: (i) $\mu=\frac{\mu_{t}}{\mu_{m}}$, the ratio of the tissue viscosity $\mu_{t}$ to the matrix

114 viscosity $\mu_{m}$, (ii) $j=\frac{\tau_{\mathrm{g}}}{\tau_{\mathrm{t}}}$, the ratio of the constant timescale to add one cell to the tissue in the

115 absence to stress, $\tau_{\mathrm{g}}$, and the varying timescale to add one cell to the confined tissue in the

116 presence of stress, $\tau_{\mathrm{t}}$ and (iii) $A=\frac{\tau_{\mathrm{a}}}{\tau_{\mathrm{m}}}$ the ratio of the cell activity timescale $\tau_{\mathrm{a}}=\frac{\tau_{\mathrm{g}}}{\epsilon} M$ where $M$

117 is the effective motility and $\epsilon$ is the strength of cell-cell adhesion, and the matrix relaxation

118 timescale, $\tau_{\mathrm{m}}=\frac{\mu_{\mathrm{m}}}{G^{\prime}}$, where $G^{\prime}$ is the shear (storage) modulus of the matrix. In our experiments,

$119 \tau_{g}$ is $\sim 30$ s for MCF10A, if one starts with the 2000 cells used in our studies, ${ }^{41}$ which is in the range

120 of our matrices stress relaxation times ( $\sim 30-350$ s). It is known that increasing the mechanical

121 stress prevents division, ${ }^{42,43}$ which will lead to a slower rate of addition of cells to the tissue $\left(\tau_{\mathrm{t}}\right)$ 
and a smaller scaled cell flux $j$. Each of these dimensionless parameters can be large or small (relative to unity) and plays a role in controlling morphological stability of the growing tissue.

Systems with low $\mu$ correspond to relatively viscous matrices, while those with high $\mu$ correspond

126

127

128 to occur both in simulations and experiments when tissue spheroid diameter was $\sim 10$ a.

to matrices that are relatively fluid. When the scaled cell flux $j$ is small, the pressure due to the growing tissue is not large enough to create fingers in the matrix, while when $j$ is large, branching likely arises as cells actively intrude into the matrix. Finally, systems with low values of $A$ correspond to matrices that mechanically relax very slowly, while systems with high values of $A$ correspond to matrices that relax very quickly. In our experiments and simulations, the ratio $\mu=\frac{\mu_{t}}{\mu_{m}} \in[0.001-2], \tau_{\mathrm{a}} \in[7-54] \mathrm{s}$, while $\tau_{\mathrm{m}} \in[1-350] \mathrm{s}$, so that the ratio $A=\frac{\tau_{\mathrm{a}}}{\tau_{\mathrm{m}}} \in[0.1-100]$, and finally with spheroid sizes $\mathrm{R} \sim 100 \mu \mathrm{m}$, and proliferative tissue timescale $\tau_{\mathrm{t}} \sim[4-500] s$, the ratio $j=\frac{\tau_{\mathrm{g}}}{\tau_{\mathrm{t}}} \in[0.002-0.25]$.

We start our simulations within this framework with a spherical ball of cells that is loosely packed within a viscoelastic matrix, and then allow the cells to divide and push each other into the matrix, straining it. To determine when divisions are energetically favorable, we use a MetropolisHastings algorithm ${ }^{44}$. Depending on the rheology of the matrix, this can either cause (i) the matrix to break, flow and be remodeled even as tissue cells form finger-like protrusions, or (ii) the matrix to respond purely elastically by straining, but not breaking, thus preventing the tissue cells from further division and maintaining a spherical boundary with the matrix. Indeed, as we decrease the relaxation time scale making the matrix behave more like a liquid (i.e. making $A=\frac{G \prime}{\mu_{\mathrm{m}}} \tau_{\mathrm{a}}$ large by decreasing $\mu_{\mathrm{m}}$ ) we see the appearance of an interfacial morphological instability (Fig. $2 \mathrm{~b}-\mathrm{d}$ and Video S2), in accordance with findings of experiments (Fig. 1). Notably, instabilities were found Additionally, when cell motility was reduced (by changing $M$ ), the model predicts that tissues 
147 growing in matrices would be unable to grow, break symmetry or form branches (Fig. $2 e$ and

148 Extended Data Fig. 4a,b).

To test these predictions, we first carried out experiments using matrices without cell adhesion ligands, as cell adhesion and thus motility would be lost in this condition $\left(A=\frac{G^{\prime}}{\mu_{\mathrm{m}}} \tau_{\mathrm{a}} \sim 0\right)$. Tissues were found to grow slowly, in a morphologically stable manner (Fig. 2f, Extended Data Fig. 4c and Video S3). Next, potential mechanisms driving tissue motility and proliferation at the cellular scale were explored. Cell motility can be regulated by: 1) cells pulling on the matrix via contractile forces generated by acto-myosin interactions involving ROCK and Non-Muscle Myosin II, 2) cells pushing on the matrix via protrusions created by Rac1 or Arp2/3 activity or 3) ion channelmediated changes ${ }^{45}$ (Fig. $2 \mathrm{~g}$ ). Only the inhibition of Rac1 or, the Rac1 pathway downstream molecule, Arp2/3 by pharmacological inhibitors (NSC23766 and CK666, respectively) inhibited tissue growth (Fig.2h and Extended Data Fig. 4d,e), in accordance with our model predictions. This finding indicates that cells generate space for division and migration by pushing on the matrix. Consistent with this, when the rate of cell proliferation in the model was inhibited $\left(j=\frac{\tau_{\mathrm{g}}}{\tau_{\mathrm{t}}} \sim 0\right)$ simulations predicted tissue growth and instability would be dramatically diminished (Fig. 2i, 163 Extended Data Fig. 5a,b and Video S4). Experiments in which cell proliferation was inhibited confirmed this prediction (Fig. 2j and Extended Data Fig. 5c). Further, the model predicts that for cells in an elastic matrix, cell division would be spatially confined to the boundary between the growing tissue and the substrate, but for cells in a viscoelastic matrix, the divisions would be more

167 broadly distributed throughout growing tissues (Fig. 2k and Extended Data Fig. 6). Experimental 168 analysis of the spatial distribution of proliferating cells in elastic versus viscoelastic matrices 169 confirmed these predictions as well (Fig. 2k and Extended Data Fig. 6). Altogether, these results 170 show that cell motility and proliferation, both of which are regulated by the viscoelasticity of the 171 matrix, control tissue spatio-temporal organization and morphogenesis. 
172 After having considered the role of matrix viscoelasticity and cell proliferation on tissue

173 organization, we now turn to adapt our computational model to include the experimentally known

174 role that links an increase in matrix stiffness with an increase in cell motility ${ }^{46}$. We assume a

175 minimal model for this, via the relation $M \propto G^{\prime}$ (Fig.3a). Simulations with this additional

176 assumption in the model predicted that tissue morphological instability would be enhanced with

177 an increase in the modulus of the matrix $G^{\prime}$ in viscoelastic matrices (making $A=\frac{G^{\prime}}{\mu_{\mathrm{m}}} \tau_{\mathrm{a}}$ large), but

178 there would not be a significant impact in more elastic matrices (Fig.3b-d, Extended Data Fig.7

179 and Video S5). To validate these simulations experimentally, the previously developed matrices

180 were modified to change their modulus $G^{\prime}$ (by changing crosslinking to yield

$181 G^{\prime} \sim 400,1700$ and $500 \mathrm{~Pa}$ ) and independently controlling the relaxation time (by changing the

182 molecular weight of alginate) and thus make the matrix more or less viscoelastic (Fig. 3e and

183 Extended Data Fig.8). In low viscosity matrices, i.e., large $A=\frac{G^{\prime}}{\mu_{\mathrm{m}}} \tau_{\mathrm{a}}$, the increase in the modulus

$184 G^{\prime}$ resulted in greater tissue growth and branching, as predicted (Fig.3f,g). To further determine

185 if these differential responses were again mediated by cell motility and proliferation, in silico

186 predictions of this model were compared to in-vitro studies performed under similar conditions.

187 As predicted by the model, inhibition of cell motility by inhibition of Rac1 and Arp2/3 complex led

188 to a greater impact on tissue growth in stiff matrices, i.e. large $A=\frac{G^{\prime}}{\mu_{\mathrm{m}}} \tau_{\mathrm{a}}$ rather than soft

189 viscoelastic matrices, i.e. small $A=\frac{G \prime}{\mu_{\mathrm{m}}} \tau_{\mathrm{a}}$ (Fig. 3h,I, Extended Data Fig.9 and Video S6). Both

190 simulations and experiments revealed that cell division increased with stiffness both in elastic and

191 viscoelastic matrices although significantly more in viscoelastic matrices (Fig.3j,k and Extended

192 Data Fig.10). The significant increase in cell flux $j$ with modulus $G^{\prime}$ in the viscoelastic matrices

$193\left(A=\frac{G \prime}{\mu_{\mathrm{m}}} \tau_{\mathrm{a}}\right.$ is large $)$ emerges from the increase in motility $M^{46}$. When cell proliferation is inhibited,

194 the simulations show that tissues do not grow (Extended Data Fig.10 and Video S7). 
195 Having studied the emergence of an active scaled cell flux from motility $M\left(\right.$ with $j=\frac{\tau_{\mathrm{g}}}{\tau_{\mathrm{t}}} \sim O(1)$ ),

196 we turn to passively inject a cell flux to the tissue (making $j=\frac{\tau_{\mathrm{g}}}{\tau_{\mathrm{t}}} \gg 1$ ) to examine the role of

197 passive tissue pressure, known to regulate tissue growth ${ }^{12,41}$, on morphological stability. To

198 achieve this, we developed a microfluidic system where cells were injected at a constant rate into

199 the tissue, displacing the matrix (Extended Data Fig.11). We find that tissues break symmetry and

200 branch out into elastic matrices but are unable to break symmetry when the matrix is viscoelastic,

201 consistent with our simulations that show a similar response (Extended Data Fig.11, 12, 13c and

202 Video S8). The morphological instability occurring in this cell flux driven situation is similar to the

203 Saffman-Taylor instability in hydrodynamics and its elastic analog ${ }^{32,33}$, wherein a low viscosity

204 (low stiffness) material forms branches when driven into a high viscosity (high stiffness) material

205 in a confined geometry. Altogether, our simulations and experiments show that the tissue-matrix

206 interface becomes morphologically unstable when the matrix is viscoelastic and can easily relax

207 in response to stresses, or when the tissue proliferative pressure is high in more elastic matrices.

208 We summarize these results in a morphological phase diagram that quantifies the stability of the

209 growing front shown in Fig. 3I and Extended Data Fig.13.

\section{Matrix viscoelasticity also regulates intestinal organoid patterning}

211 To explore the generality of these findings as captured by the phase diagram in Fig. 3I, we decided

212 to explore the impact of matrix viscoelasticity in a synthetic context associated with the in-vitro

213 growth and development of self-organizing intestinal organoids. When Lgr5+ stem cells are

214 emplaced in a complex, laminin-rich extracellular matrix termed Matrigel, they develop into

215 complex three-dimensional structures containing all cell-types present in adult intestine, and

216 mimic intestinal tissue organization ${ }^{1,8}$. To allow for a comparison with the published literature, we

217 modified our alginate matrix system to enable incorporation of Matrigel (Fig. 4a), while still

218 allowing independent control over gel stiffness and viscoelasticity ${ }^{31}$. The interpenetrating 
219 networks of two different stiffness $\left(G^{\prime} \sim 0.5 \mathrm{kPa}\right.$ and $\left.1.5 \mathrm{kPa}\right)$ allowed for both elastic and viscoelastic

220 matrices (Fig. 4b). As previously described ${ }^{17,47,48}$, organoids growing in elastic matrices exhibited

221 slow expansion and were morphologically stable. In contrast, intestinal organoids grew rapidly,

222 broke symmetry and formed branches when within viscoelastic matrices (Fig. 4c-e and Extended

223 Data Fig.14). Apart from demonstrating tissue morphological instability, organoids in viscoelastic

224 substrates exhibited cell patterning and differentiation representative of intestinal development

225 (Fig. 4f,g). Matrix viscoelasticity favored the generation of high curvature tissue regions that

226 concentrated Lgr5+ stem cells, consistent with past reports on the impact of curvature on

227 differentiation ${ }^{18}$. To determine if organoid spatio-temporal organization was regulated by internal

228 pressure generated inside organoid lumens, as previously reported with other systems ${ }^{14,15,49}$,

229 organoids were pharmacologically treated to impair the function of $\mathrm{Na}+\mathrm{K}+$ ATPase pumps and

230 block fluid influx ${ }^{14,50}$. No significant differences in organoid morphology or patterning in

231 viscoelastic substrates were noted (Extended Data Fig. 15). To further test the ability of

232 viscoelasticity to control organ growth, organoid development was monitored in matrices of

233 varying stiffness. The percentage of Lgr5+ organoids and number of colonies were higher in

234 viscoelastic matrices rather than elastic matrices, independent of $G^{\prime}$ (Fig.4h,i). This finding is

235 consistent with previous research as symmetry breaking and organoid development are

236 associated with a higher percentage of Lgr5+ organoids ${ }^{1}$. Increasing $G^{\prime}$ of viscoelastic matrices

237 again led to greater growth of intestinal organoids, symmetry breaking and branch formation, but

238 organoids grew more slowly and maintained their spherical symmetry in elastic matrices (Fig.4j-

239 I).

\section{Discussion}

241 Our experiments and guiding simulations demonstrate that passive matrix viscoelasticity couples

242 to cell motility and cell proliferation to drive tissue growth, symmetry breaking and branching. The

243 resulting morphology is reminiscent of interfacial instabilities in passively driven physical systems 
244 but modified fundamentally in living systems by the active processes of cell motility and cell

245 proliferation that can destabilize the interface and are relevant to a number of processes

246 including embryogenesis ${ }^{3,14}$, oncogenesis ${ }^{2,51}$, branching morphogenesis ${ }^{6,7}$, and angiogenesis.

247 Our studies of two different systems: breast epithelia and intestinal organoids, show that the

248 properties of the viscoelastic extracellular matrix relative to that of the tissue, quantified in terms

249 of three experimentally-manipulatable dimensionless parameters, emerge as regulators of spatio-

250 temporal tissue organization.

252 More broadly, our results are consistent with prior observations that the increase in ECM fluidity 253 of the mesenchyme drives normal embryonic airway branching ${ }^{52}$, and an increase in tissue fluidity 254 drives wound healing ${ }^{53}$, tissue elongation ${ }^{54}$ or neural crest development ${ }^{55}$. Furthermore, invasive branches are characterized by either an increase in matrix fluidity, as has recently been observed

256 in glioblastoma ${ }^{56,57}$, breast ${ }^{58}$ and liver cancer ${ }^{59}$ (compared to benign lesions and healthy ECM), or

257 an increase in tissue fluidity, as tumor single cells are less viscous ${ }^{60,61}$ and tumor tissues acquire 258 more liquid-like properties ${ }^{62-64}$ (e.g. EMT, unjamming). The increased expression of low molecular 259 weight hyaluronic acid in malignant tumors ${ }^{65}$ can explain the decrease in tumor ECM viscosity.

260 Our results also suggest that when tumors migrate and grow and push the stroma, this may lead 261 to the passive generation of stroma fingers in the healthy tissue, as the stroma has more liquid262 like properties than healthy tissue ${ }^{56-59}$.We can also rationalize previous apparently contradictory 263 findings that tissues maintained a stable morphology when encapsulated in synthetic materials ${ }^{66}$ 264 of increasing stiffness, while becoming unstable in natural matrices as stiffness was raised ${ }^{2,67}$ 265 (e.g. Matrigel, collagen, fibrin). From our perspective, the explanation is due to the elastic nature 266 of the synthetics that are covalently crosslinked, in contrast to the intrinsic viscoelasticity of 267 physically cross-linked natural matrices. Finally, in addition to providing a framework to 268 understand tissue morphology and organization in normal and pathological states, our study 
270 regenerative medicine and related fields.

\section{References:}

2751 Sato, T. et al. Single Lgr5 stem cells build crypt-villus structures in vitro without a

276 mesenchymal niche. Nature 459, 262-265, doi:10.1038/nature07935 (2009).

2772 Paszek, M. J. et al. Tensional homeostasis and the malignant phenotype. Cancer cell 8, 278 241-254, doi:10.1016/j.ccr.2005.08.010 (2005).

2793 Deglincerti, A. et al. Self-organization of the in vitro attached human embryo. Nature

$280 \quad 533,251-254$, doi:10.1038/nature17948 (2016).

2814 Moris, N. et al. An in vitro model of early anteroposterior organization during human

282 development. Nature 582, 410-415, doi:10.1038/s41586-020-2383-9 (2020).

$2835 \quad$ Krndija, D. et al. Active cell migration is critical for steady-state epithelial turnover in the gut. Science 365, 705-710, doi:10.1126/science.aau3429 (2019).

6 Hannezo, E. \& Simons, B. D. Multiscale dynamics of branching morphogenesis. Current opinion in cell biology 60, 99-105, doi:10.1016/j.ceb.2019.04.008 (2019).

7 Varner, V. D. \& Nelson, C. M. Cellular and physical mechanisms of branching morphogenesis. Development 141, 2750-2759, doi:10.1242/dev.104794 (2014).

8 Serra, D. et al. Self-organization and symmetry breaking in intestinal organoid development. Nature 569, 66-72, doi:10.1038/s41586-019-1146-y (2019).

9 Hannezo, E. et al. A Unifying Theory of Branching Morphogenesis. Cell 171, 242-255 e227, doi:10.1016/j.cell.2017.08.026 (2017).

10 Briggs, J. A. et al. The dynamics of gene expression in vertebrate embryogenesis at single-cell resolution. Science 360, doi:10.1126/science.aar5780 (2018).

11 Hannezo, E. \& Heisenberg, C. P. Mechanochemical Feedback Loops in Development and Disease. Cell 178, 12-25, doi:10.1016/j.cell.2019.05.052 (2019).

12 Green, J. B. \& Sharpe, J. Positional information and reaction-diffusion: two big ideas in developmental biology combine. Development 142, 1203-1211, doi:10.1242/dev.114991 (2015).

13 Kondo, S. \& Miura, T. Reaction-diffusion model as a framework for understanding biological pattern formation. Science 329, 1616-1620, doi:10.1126/science.1179047 (2010).

14 Chan, C. J. et al. Hydraulic control of mammalian embryo size and cell fate. Nature 571, 112-116, doi:10.1038/s41586-019-1309-x (2019).

15 Mosaliganti, K. R. et al. Size control of the inner ear via hydraulic feedback. Elife 8, doi:10.7554/eLife.39596 (2019).

16 Nelson, C. M. et al. Microfluidic chest cavities reveal that transmural pressure controls the rate of lung development. Development 144, 4328-4335, doi:10.1242/dev.154823 (2017).

17 Gjorevski, N. et al. Designer matrices for intestinal stem cell and organoid culture. Nature 539, 560-564, doi:10.1038/nature20168 (2016). 
Shyer, A. E., Huycke, T. R., Lee, C., Mahadevan, L. \& Tabin, C. J. Bending gradients:

19 Harris, A. R. et al. Characterizing the mechanics of cultured cell monolayers. Proceedings of the National Academy of Sciences of the United States of America 109, 16449-16454, doi:10.1073/pnas.1213301109 (2012).

20 Vining, K. H. \& Mooney, D. J. Mechanical forces direct stem cell behaviour in development and regeneration. Nature reviews. Molecular cell biology 18, 728-742, doi:10.1038/nrm.2017.108 (2017).

21 Khalilgharibi, N., Fouchard, J., Recho, P., Charras, G. \& Kabla, A. The dynamic mechanical properties of cellularised aggregates. Current opinion in cell biology 42, 113120, doi:10.1016/j.ceb.2016.06.003 (2016).

22 Ricca, B. L., Venugopalan, G. \& Fletcher, D. A. To pull or be pulled: parsing the multiple modes of mechanotransduction. Current opinion in cell biology 25, 558-564, doi:10.1016/j.ceb.2013.06.002 (2013).

23 Heisenberg, C. P. \& Bellaiche, Y. Forces in tissue morphogenesis and patterning. Cell 153, 948-962, doi:10.1016/j.cell.2013.05.008 (2013).

24 Chaudhuri, O. et al. Hydrogels with tunable stress relaxation regulate stem cell fate and activity. Nature materials 15, 326-334, doi:10.1038/nmat4489 (2016).

25 Debnath, J., Muthuswamy, S. K. \& Brugge, J. S. Morphogenesis and oncogenesis of MCF-10A mammary epithelial acini grown in three-dimensional basement membrane cultures. Methods 30, 256-268 (2003).

26 Lee, K. Y. \& Mooney, D. J. Alginate: properties and biomedical applications. Progress in polymer science 37, 106-126, doi:10.1016/j.progpolymsci.2011.06.003 (2012).

27 Rowley, J. A., Madlambayan, G. \& Mooney, D. J. Alginate hydrogels as synthetic extracellular matrix materials. Biomaterials 20, 45-53 (1999).

28 Yang, J. et al. Guidelines and definitions for research on epithelial-mesenchymal transition. Nature reviews. Molecular cell biology, doi:10.1038/s41580-020-0237-9 (2020).

29 Elosegui-Artola, A. et al. Mechanical regulation of a molecular clutch defines force transmission and transduction in response to matrix rigidity. Nature cell biology 18, 540548, doi:10.1038/ncb3336 (2016).

30 Zanconato, F., Cordenonsi, M. \& Piccolo, S. YAP/TAZ at the Roots of Cancer. Cancer cell 29, 783-803, doi:10.1016/j.ccell.2016.05.005 (2016).

31 Chaudhuri, O. et al. Extracellular matrix stiffness and composition jointly regulate the induction of malignant phenotypes in mammary epithelium. Nature materials 13, 970978, doi:10.1038/nmat4009 (2014).

32 Saffman, P. G. \& Taylor, G. I. The penetration of a fluid into a porous medium or HeleShaw cell containing a more viscous liquid. Proceedings of the Royal Society of London. Series A. Mathematical and Physical Sciences 245, 312-329, doi:doi:10.1098/rspa.1958.0085 (1958).

33 Biggins, J. S., Saintyves, B., Wei, Z., Bouchaud, E. \& Mahadevan, L. Digital instability of a confined elastic meniscus. Proceedings of the National Academy of Sciences of the United States of America 110, 12545-12548, doi:10.1073/pnas. 1302269110 (2013).

34 Montel, F. et al. Stress clamp experiments on multicellular tumor spheroids. Phys Rev Lett 107, 188102, doi:10.1103/PhysRevLett.107.188102 (2011).

35 Bogdan, M. J. \& Savin, T. Fingering instabilities in tissue invasion: an active fluid model. Royal Society Open Science 5, 181579, doi:doi:10.1098/rsos.181579 (2018).

36 Risler, T. \& Basan, M. Morphological instabilities of stratified epithelia: a mechanical instability in tumour formation. New Journal of Physics 15, 065011, doi:10.1088/13672630/15/6/065011 (2013). 
363

364

365

366

367

368

369

370

371

372

373

374

375

376

377

378

379

380

381

382

383

384

385

386

387

388

389

390

391

392

393

394

395

396

397

398

399

400

401

402

403

404

405

406

407

408

409

410

411

37 Shaebani, M. R., Wysocki, A., Winkler, R. G., Gompper, G. \& Rieger, H. Computational models for active matter. Nature Reviews Physics 2, 181-199, doi:10.1038/s42254-0200152-1 (2020).

38 Marchetti, M. C. et al. Hydrodynamics of soft active matter. Reviews of Modern Physics 85, 1143-1189, doi:10.1103/RevModPhys.85.1143 (2013).

39 Mallory, S. A., Šarić, A., Valeriani, C. \& Cacciuto, A. Anomalous thermomechanical properties of a self-propelled colloidal fluid. Physical Review E 89, 052303, doi:10.1103/PhysRevE.89.052303 (2014).

40 Berthier, L. \& Kurchan, J. Non-equilibrium glass transitions in driven and active matter. Nature Physics 9, 310-314, doi:10.1038/nphys2592 (2013).

41 Araujo, A. R., Gelens, L., Sheriff, R. S. \& Santos, S. D. Positive Feedback Keeps Duration of Mitosis Temporally Insulated from Upstream Cell-Cycle Events. Mol Cell 64, 362-375, doi:10.1016/j.molcel.2016.09.018 (2016).

42 Uroz, M. et al. Regulation of cell cycle progression by cell-cell and cell-matrix forces. Nature cell biology 20, 646-654, doi:10.1038/s41556-018-0107-2 (2018).

43 Delarue, M. et al. Compressive Stress Inhibits Proliferation in Tumor Spheroids through a Volume Limitation. Biophysical journal 107, 1821-1828, doi:https://doi.org/10.1016/i.bpi.2014.08.031 (2014).

44 Hastings, W. K. Monte Carlo Sampling Methods Using Markov Chains and Their Applications. Biometrika 57, 97-109, doi:10.2307/2334940 (1970).

45 Hung, W. C. et al. Confinement Sensing and Signal Optimization via Piezo1/PKA and Myosin II Pathways. Cell Rep 15, 1430-1441, doi:10.1016/j.celrep.2016.04.035 (2016).

46 Ulrich, T. A., de Juan Pardo, E. M. \& Kumar, S. The mechanical rigidity of the extracellular matrix regulates the structure, motility, and proliferation of glioma cells. Cancer research 69, 4167-4174, doi:10.1158/0008-5472.CAN-08-4859 (2009).

47 Hushka, E. A., Yavitt, F. M., Brown, T. E., Dempsey, P. J. \& Anseth, K. S. Relaxation of Extracellular Matrix Forces Directs Crypt Formation and Architecture in Intestinal Organoids. Advanced Healthcare Materials 9, 1901214, doi:10.1002/adhm.201901214 (2020).

48 Cruz-Acuna, R. et al. Synthetic hydrogels for human intestinal organoid generation and colonic wound repair. Nature cell biology 19, 1326-1335, doi:10.1038/ncb3632 (2017).

49 Dasgupta, S., Gupta, K., Zhang, Y., Viasnoff, V. \& Prost, J. Physics of lumen growth. Proceedings of the National Academy of Sciences of the United States of America 115, E4751-E4757, doi:10.1073/pnas.1722154115 (2018).

50 Violette, M. I., Madan, P. \& Watson, A. J. Na+/K+ -ATPase regulates tight junction formation and function during mouse preimplantation development. Developmental biology 289, 406-419, doi:10.1016/j.ydbio.2005.11.004 (2006).

51 Friedl, P., Locker, J., Sahai, E. \& Segall, J. E. Classifying collective cancer cell invasion. Nature cell biology 14, 777-783, doi:10.1038/ncb2548 (2012).

52 Spurlin, J. W. et al. Mesenchymal proteases and tissue fluidity remodel the extracellular matrix during airway epithelial branching in the embryonic avian lung. Development 146, doi:10.1242/dev.175257 (2019).

53 Tetley, R. J. et al. Tissue fluidity promotes epithelial wound healing. Nature Physics 15, 1195-1203, doi:10.1038/s41567-019-0618-1 (2019).

54 Mongera, A. et al. A fluid-to-solid jamming transition underlies vertebrate body axis elongation. Nature 561, 401-405, doi:10.1038/s41586-018-0479-2 (2018).

55 Kuriyama, S. et al. In vivo collective cell migration requires an LPAR2-dependent increase in tissue fluidity. The Journal of cell biology 206, 113-127, doi:10.1083/jcb.201402093 (2014). 
56 Streitberger, K. J. et al. High-resolution mechanical imaging of glioblastoma by multifrequency magnetic resonance elastography. PloS one 9, e110588, doi:10.1371/journal.pone.0110588 (2014).

57 Streitberger, K. J. et al. How tissue fluidity influences brain tumor progression. Proceedings of the National Academy of Sciences of the United States of America 117, 128-134, doi:10.1073/pnas.1913511116 (2020).

58 Sinkus, R. et al. Viscoelastic shear properties of in vivo breast lesions measured by MR elastography. Magnetic resonance imaging 23, 159-165, doi:10.1016/j.mri.2004.11.060 (2005).

59 Shahryari, M. et al. Tomoelastography distinguishes non-invasively between benign and malignant liver lesions. Cancer research, canres.2150.2019, doi:10.1158/00085472.Can-19-2150 (2019).

60 Nematbakhsh, Y., Pang, K. T. \& Lim, C. T. Correlating the viscoelasticity of breast cancer cells with their malignancy. Convergent Science Physical Oncology 3, 034003, doi:10.1088/2057-1739/aa7ffb (2017).

61 Ketene, A. N., Schmelz, E. M., Roberts, P. C. \& Agah, M. The effects of cancer progression on the viscoelasticity of ovarian cell cytoskeleton structures. Nanomedicine: Nanotechnology, Biology and Medicine 8, 93-102, doi:https://doi.org/10.1016/i.nano.2011.05.012 (2012).

62 Palamidessi, A. et al. Unjamming overcomes kinetic and proliferation arrest in terminally differentiated cells and promotes collective motility of carcinoma. Nature materials 18, 1252-1263, doi:10.1038/s41563-019-0425-1 (2019).

63 Han, Y. L. et al. Cell swelling, softening and invasion in a three-dimensional breast cancer model. Nature Physics 16, 101-108, doi:10.1038/s41567-019-0680-8 (2020).

64 Staneva, R. et al. Cancer cells in the tumor core exhibit spatially coordinated migration patterns. Journal of cell science 132, doi:10.1242/jcs.220277 (2019).

65 Monslow, J., Govindaraju, P. \& Puré, E. Hyaluronan - A Functional and Structural Sweet Spot in the Tissue Microenvironment. Frontiers in immunology 6 , doi:10.3389/fimmu.2015.00231 (2015).

66 Taubenberger, A. V. et al. 3D Microenvironment Stiffness Regulates Tumor Spheroid Growth and Mechanics via p21 and ROCK. Advanced Biosystems 3, 1900128, doi:10.1002/adbi.201900128 (2019).

67 Kumar, S. \& Weaver, V. M. Mechanics, malignancy, and metastasis: the force journey of a tumor cell. Cancer metastasis reviews 28, 113-127, doi:10.1007/s10555-008-9173-4 (2009). 
a
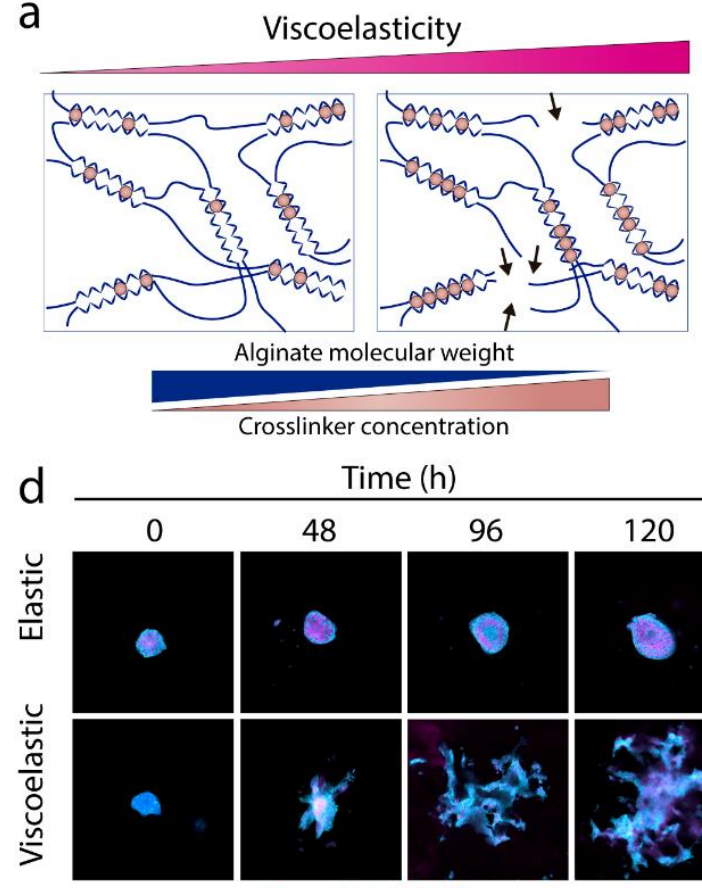

g
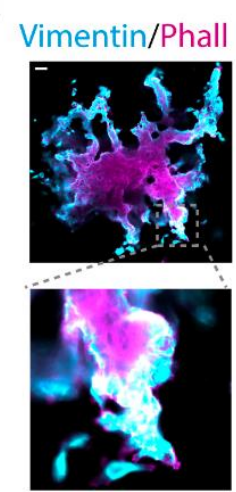

$\mathrm{k}$

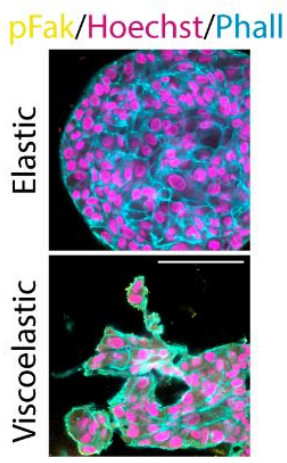

Time (h)
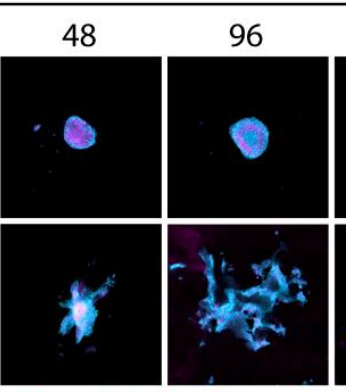

Hoechst/Phall

$\mathrm{h}$

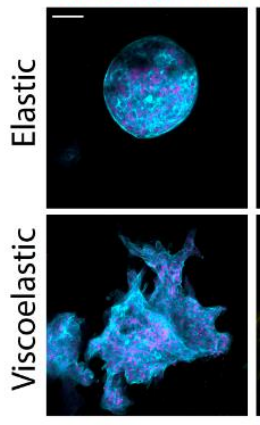

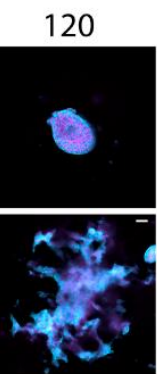

Hoechst/Phall Cytokeratin14
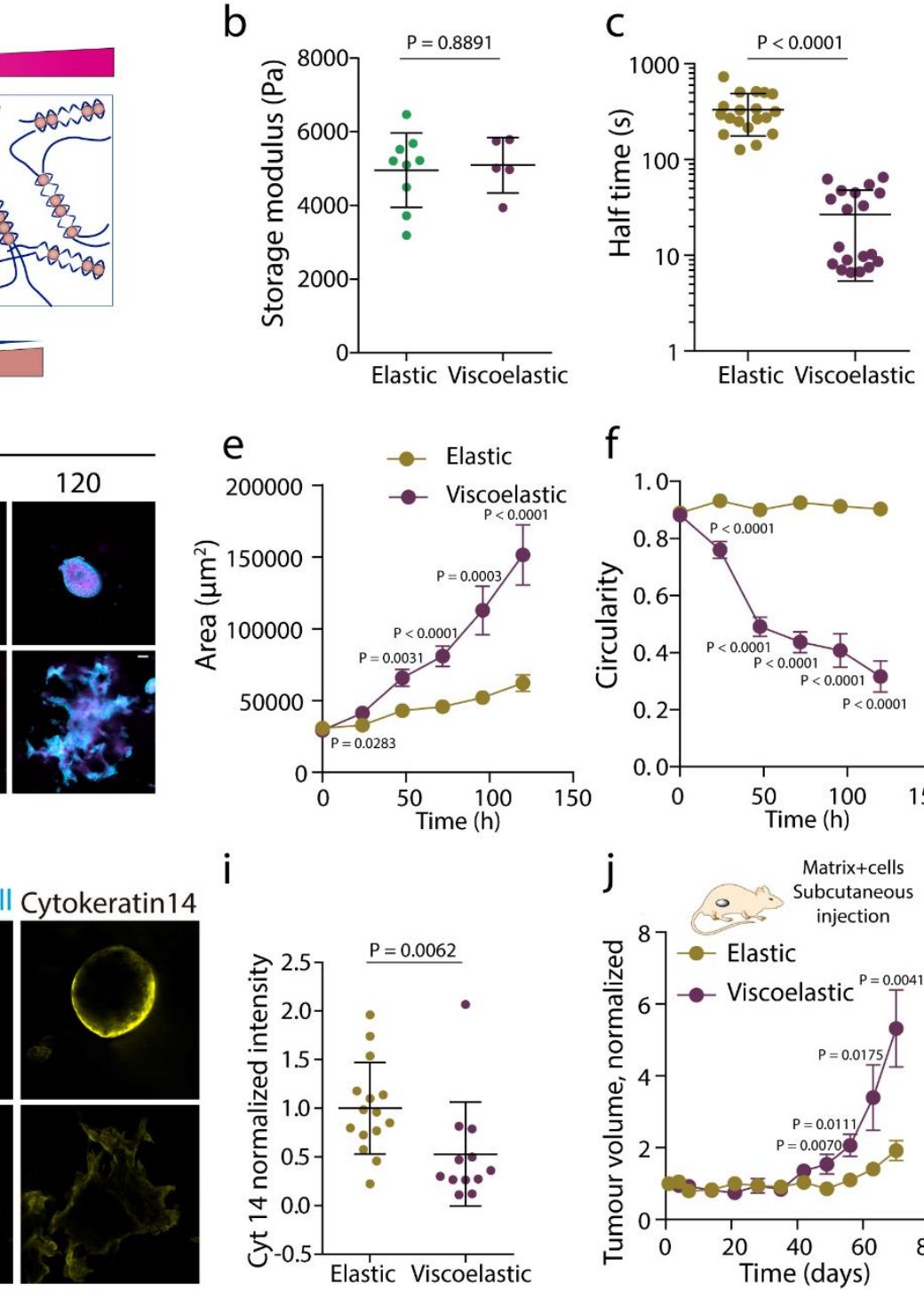

f
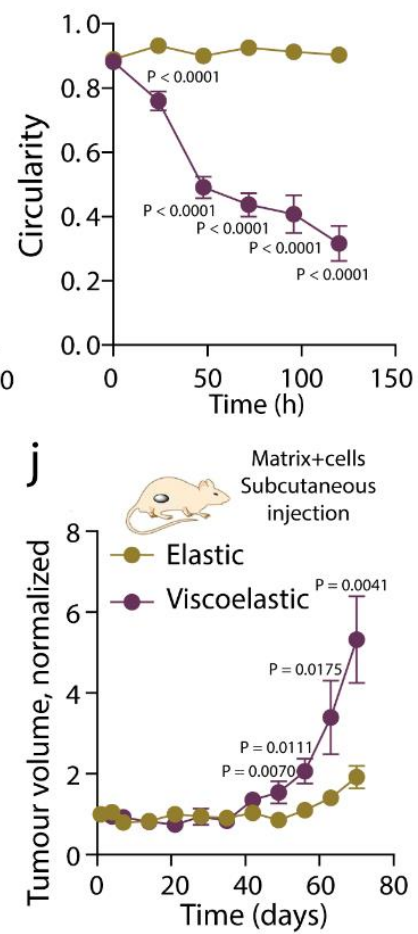

Viscoelastic
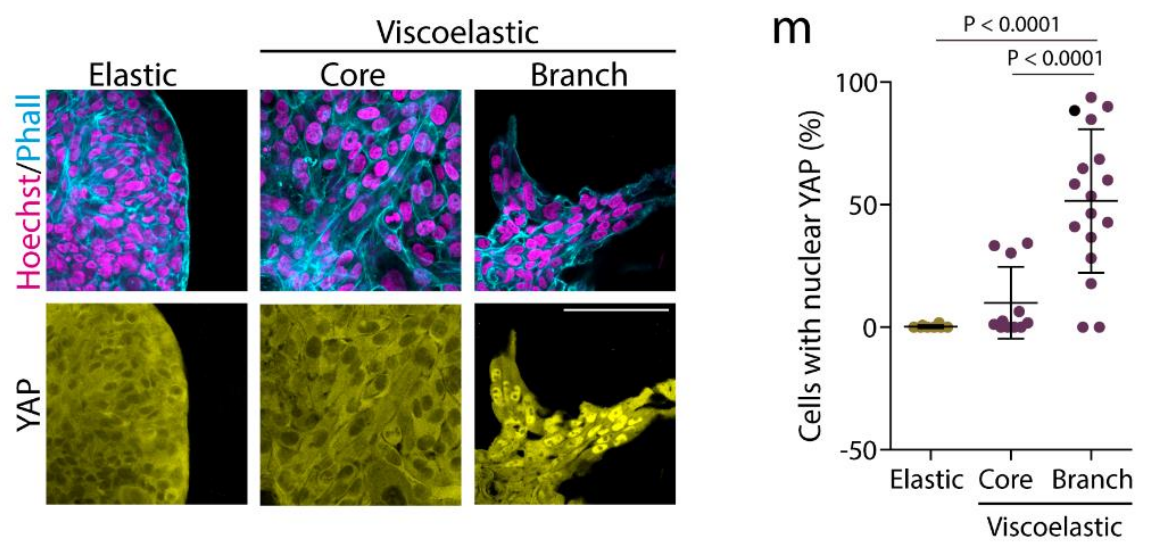
a, Schematic demonstrating how simultaneously changing the polymer molecular weight and extent of crosslinking allows for constant gel stiffness but altered viscoelastic properties. $\mathbf{b}$, Quantification of the storage modulus of resulting alginate hydrogels ( $n=5,9$ gels per condition). Statistical analysis was performed using two-sided U Mann-Whitney test. c, Quantification of the timescale at which an initially applied stress is relaxed to half its original value ( $n=19$ gels per condition). Statistical analysis was performed using Mann-Whitney U-test. d, Examples of growth of MCF10A spheroids in elastic versus viscoelastic hydrogels over 5 days. Phalloidin in cyan, Hoechst in magenta. e-f, Quantification of the spheroids area (e) and circularity (f), respectively (error bars, s.e.m). n=19-43 spheroids/condition/day. Statistical analysis was performed using Kruskal-Wallis test followed by post hoc Dunn's test. g. Examples of vimentin, phalloidin and hoechst stainings in spheroids growing in viscoelastic gels. Insets shows a spheroid branch. $\mathbf{h}$. Examples of phalloidin, Hoechst (left) and cytokeratin 14 (right) stainings in spheroids in viscoelastic and elastic hydrogels. Phalloidin in cyan, Hoechst in magenta and cytokeratin 14 in yellow. i, Quantification of average cytokeratin 14 intensity of the outer ring of spheroids. Elastic spheroids average intensity is normalized to $1 . n=12,15$ spheroids per condition. Statistical analysis was performed using Mann-Whitney U-test. j. Quantification of the tumor volume in mice injected in day 0 with viscoelastic and elastic hydrogels containing MDA-MB231 breast epithelial cells (error bars, s.e.m). k, Representative examples of phosphorylated FAK, phalloidin and Hoechst stainings in MCF10A celll spheroids growing in elastic and viscoelastic gels. pFAK in yellow, phalloidin in cyan and hoechst in magenta. $\mathbf{h}$. Representative examples of phalloidin, Hoechst (upper row) and YAP (lower row) stainings of spheroids in elastic and viscoelastic gels (spheroids core cells and branch leader cells). i. Quantification from stainings of the percentage of cells with nuclear YAP per image for the indicated regions ( $n=8,11,17$ images per condition). 
bioRxiv preprint doi: https://doi.org/10.1101/2022.01.19.476771; this version posted January 20,2022. The copyright holder for this preprint (which was not certified by peer review) is the author/funder, who has granted bioRxiv a license to display the preprint in perpetuity. It is made available under aCC-BY-NC-ND 4.0 International license.

a
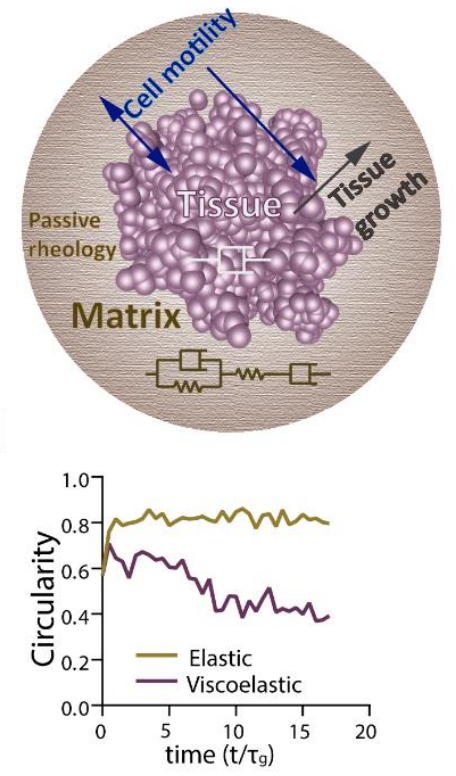

9
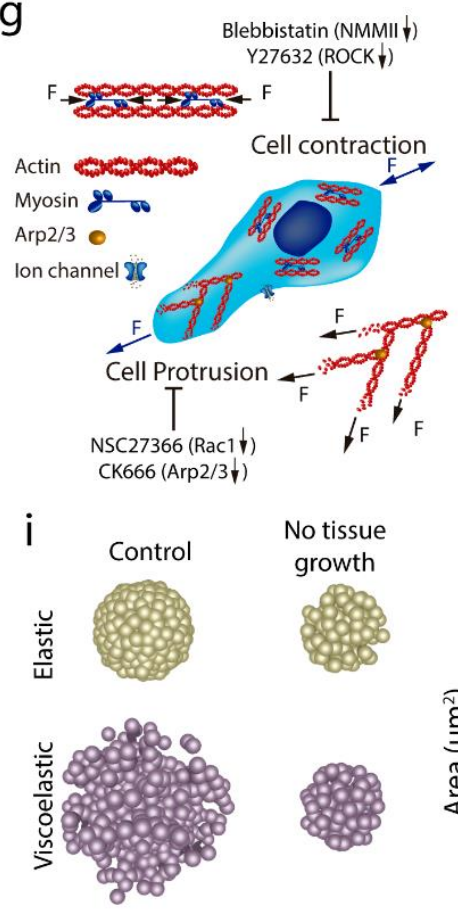

No tissue
growth
888

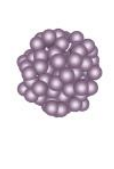

b
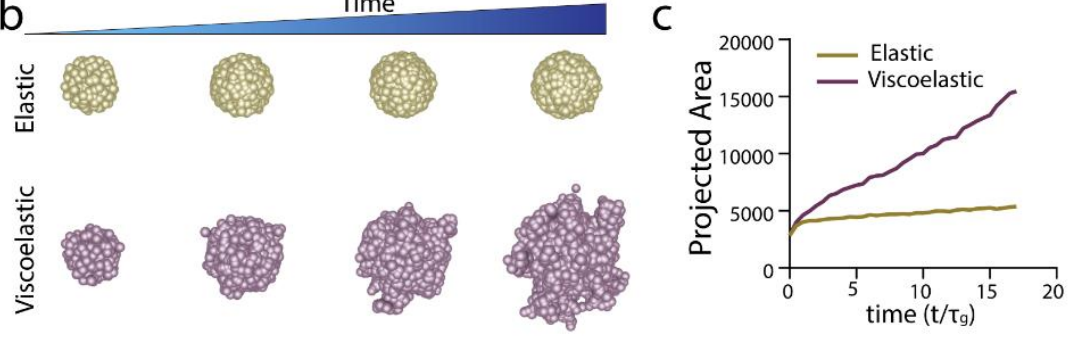

e

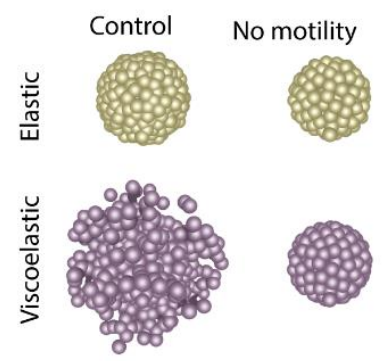

h

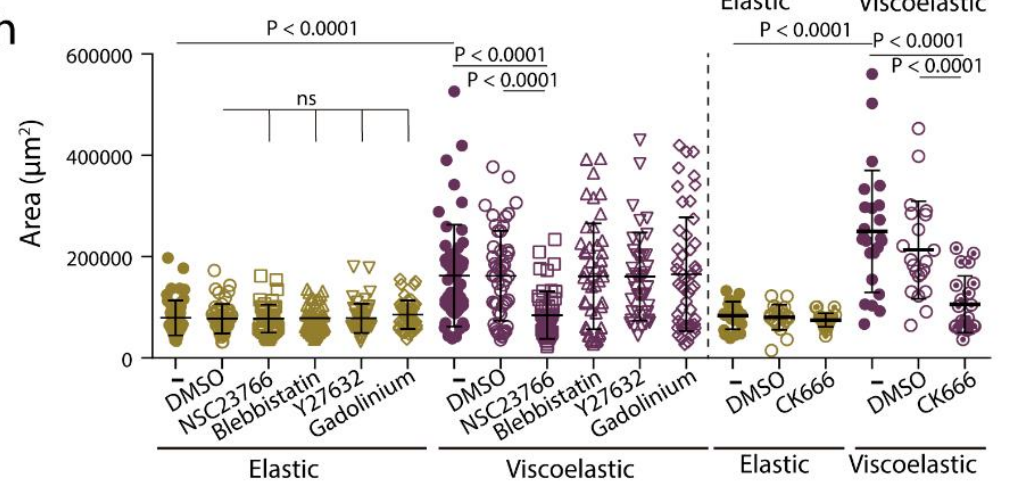

$\mathrm{k}$
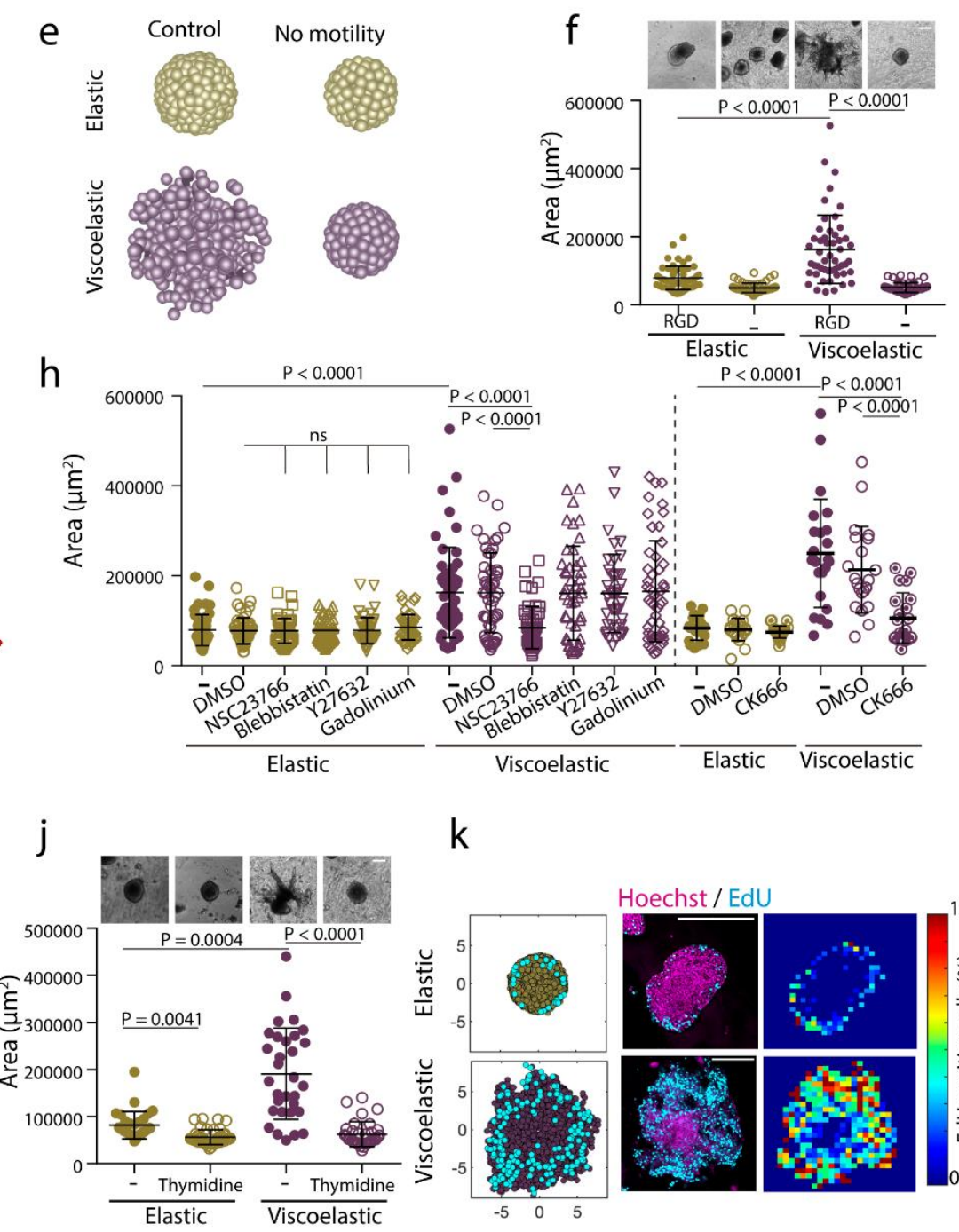

479 Figure 2: 3D theoretical model predicts that spheroids-material physical interaction

481 a, Schematic depicting the theoretical physical model of tissue growth in a passive viscoelastic 
tuned independently. b, Examples of simulated tissue growth in elastic matrices (top row) as versus viscoelastic matrices (lower row). c-d, Quantification from the simulations of the projected area and circularity of the spheroids, respectively, over time. e, Model prediction with inhibition of cell motility. $\mathbf{f}$, Representative experimental examples (upper row) and quantification of spheroid's area (lower row) in hydrogels after 5 days in gels with and without cell adhesive ligand RGD. $n=52,52,51,54$ spheroids per condition. Statistical analysis was performed using Kruskal-Wallis test followed by post hoc Dunn's test. g, Schematic showing the inhibitors used to affect cell motility:1) Blebbistatin and Y27632 affect actomyosin cytoskeleton by affecting non-muscle myosin II and ROCK, respectively; 2) Cell protrusion is affected by NSC23766 and CK666 that affect Rac1 and Arp2/3 complex, respectively; and 3) gadolinium affects ion channels. h, Quantification of spheroid area in hydrogels after 5 days in the presence of the indicated inhibitors. $\mathrm{n}=52,50,51,51,51,50,51,50,51,46,41,51,21,21,24,20,21,25$ spheroids per condition. Statistical analysis was performed using Kruskal-Wallis test followed by post hoc Dunn's test. i, Model predictions with tissue growth inhibition. j, Representative experimental examples and quantification of the spheroid's area without or with the presence of thymidine to inhibit cell proliferation. $n=52,53,51,53$ spheroids per condition. Statistical analysis was performed using Kruskal-Wallis test followed by post hoc Dunn's test. k, Model predictions and experimental results for the numbers and distributions of proliferating cells across spheroids in elastic (upper row) and viscoelastic gels (lower row): left, model predictions of localization of cell division (cyan) from a section of a spheroid; center, representative examples of experimental spheroids showing viscoelastic gels; right, colormaps of experimental image (center) showing the local percentage 505 of EdU positive cells across the spheroid. $n=3,4$ spheroids per condition. All data are mean \pm s.d., 506 all scale bars are $200 \mu \mathrm{m}$. 
bioRxiv preprint doi: https://doi.org/10.1101/2022 01.19.476771; this version posted January 20, 2022. The copyright holder for this preprint (which was not certified by peer review) is the author/funder, who has granted bioRxiv a license to display the preprint in perpetuity. It is made available under aCC-BY-NC-ND 4.0 International license.

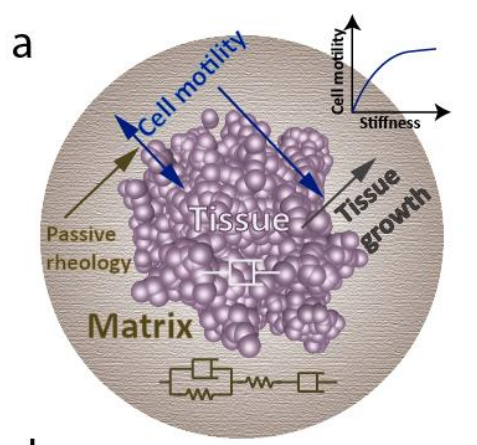

d

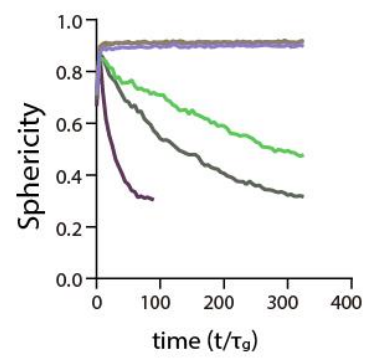

g
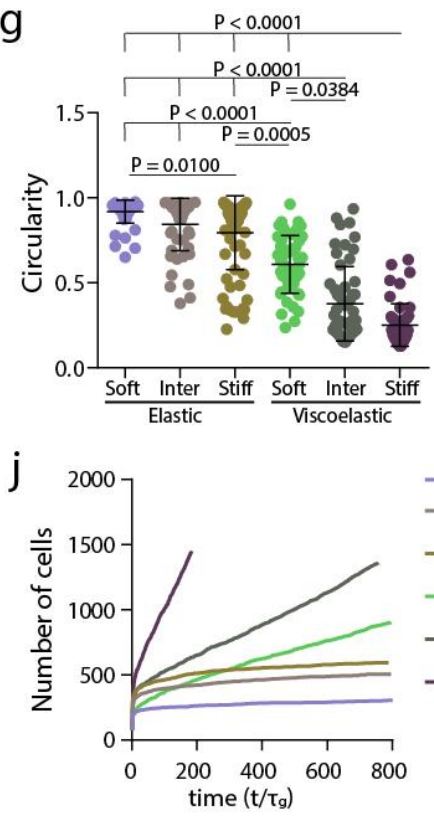

k

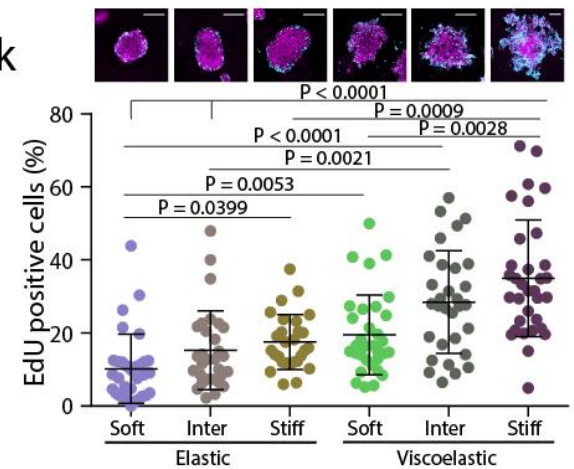

e

$\mathrm{h}$
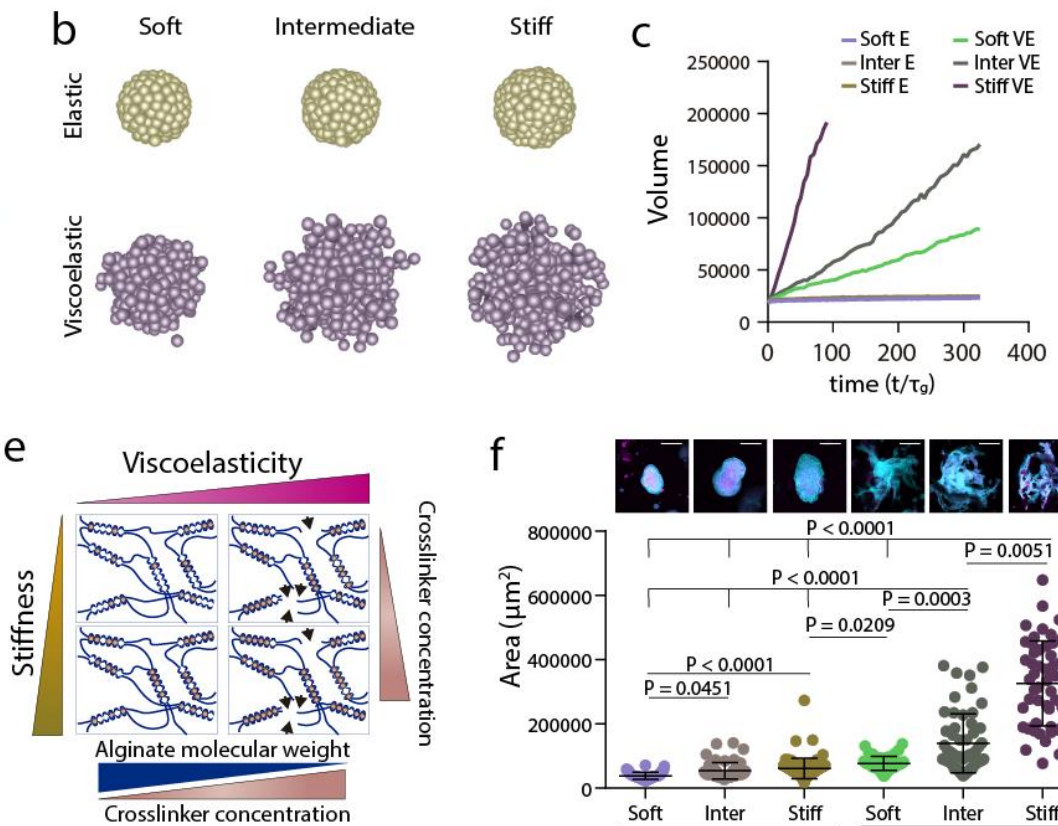

f
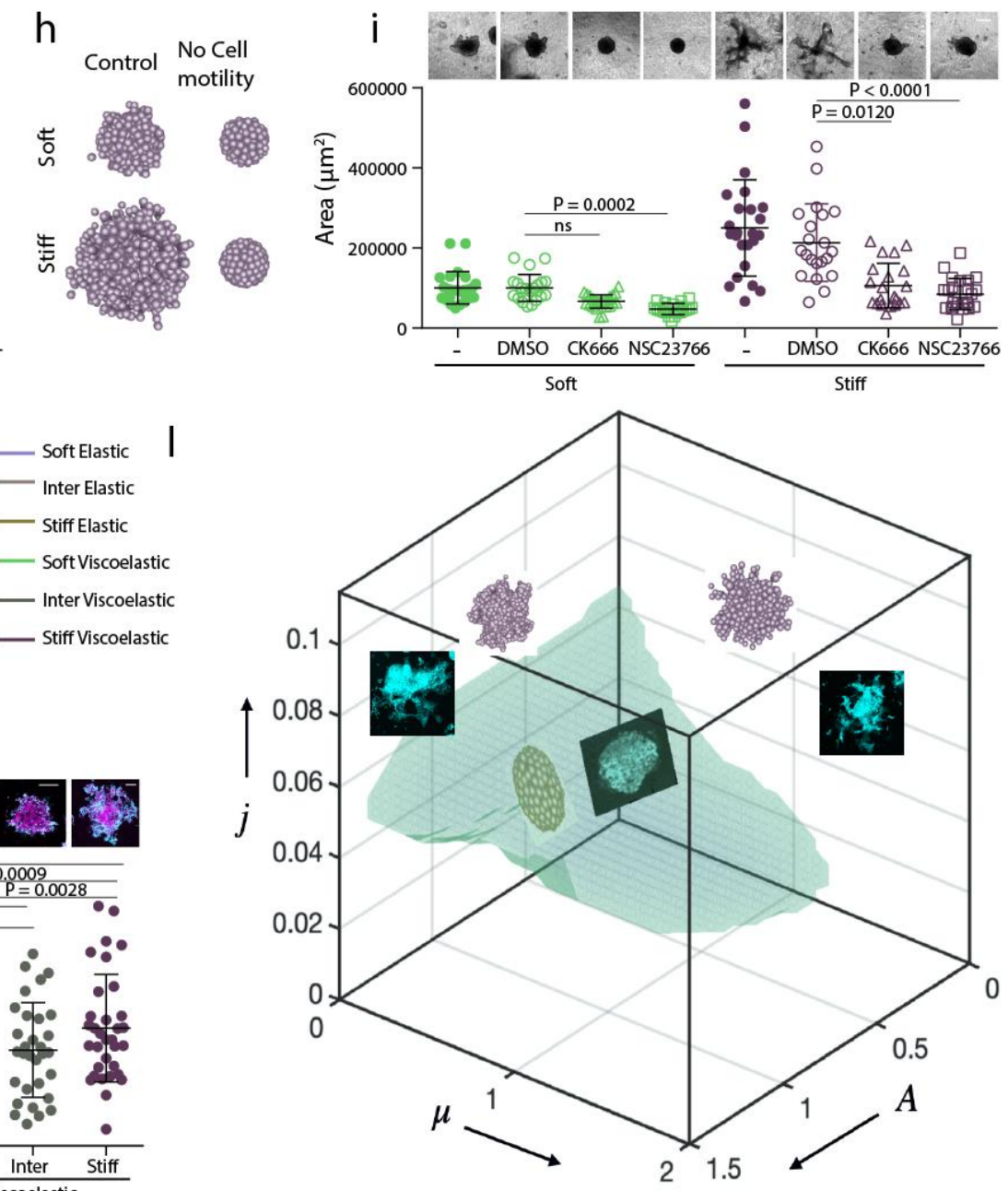
Figure 3. Stiffness intersects with matrix viscoelasticity to regulate growth and branching.

509 a, To incorporate the matrix stiffness dependence on the tissue property, now the active motility of the tissue is an increasing function of the matrix stiffness. Which makes the active motility a dependent parameter and in turn it also affects the tissue growth. b-d, 3D final timepoint simulation images (b), projected area (c) and circularity (d) evolution over time of spheroids in increasingly stiff elastic and viscoelastic gels. e, Stiffness of experimental matrices was modified by further altering the extent of crosslinking in both elastic and viscoelastic gels. $\mathbf{f}$, Representative experimental examples (upper row) and quantification of spheroid area (lower row) after 5 days in elastic and viscoelastic matrices of increasing stiffness. $n=63,55,84,50,55,50$ spheroids per condition. Statistical analysis was performed using Kruskal-Wallis test followed by post hoc Dunn's test. g, Quantification of spheroid circularity after 5 days in elastic and viscoelastic matrices of increasing stiffness. $n=63,55,84,50,55,50$ spheroids per condition. Statistical analysis was performed using Kruskal-Wallis test followed by post hoc Dunn's test. $\mathbf{h}$, Representative model simulation results when cell motility is eliminated in stiff viscoelastic matrices compared to soft viscoelastic matrices. i, Representative experimental examples (upper row) and quantification of spheroid's area (lower row) after 5 days in soft and stiff viscoelastic matrices with Rac1 (NSC23766) and Arp2/3 (CK666) inhibitors. $n=25,22,27,21,24,21,21,24$ spheroids per condition. Statistical analysis was performed using Kruskal-Wallis test followed by post hoc Dunn's test. j, Model predictions for cell proliferation in spheroids of increasing stiffness for both elastic and viscoelastic gels. k, Representative experimental examples (upper row) and quantification of the percentage of EdU positive cells in a spheroid (lower row) after 5 days in elastic and viscoelastic gels of increasing stiffness. $n=32,30,28,33,31,33$ spheroids per condition. Statistical analysis was performed using Kruskal-Wallis test followed by post hoc Dunn's test. All data are mean \pm s.d., all scale bars are $200 \mu \mathrm{m}$. I, phase diagram. Simulations predict, and experiments confirm that regions of tissue growth stability and instability can be predicted based on the values of three dimensionless variables. When the scaled proliferation pressure $j=\frac{\tau_{\mathrm{g}}}{\tau_{\mathrm{t}}} \ll$ 1, the tissue grows as a stable spheroid (Fig. 2i,j and Extended Data Fig. 9, 10, 13b). Additionally, when the scaled matrix relaxation time $A=\frac{\tau_{\mathrm{a}}}{\tau_{\mathrm{m}}} \ll 1$, the tissue remains spheroidal and is morphologically stable as long as the scaled proliferation pressure $j=\frac{\tau_{\mathrm{g}}}{\tau_{\mathrm{t}}} \sim O$ (1) (top panel of Fig.1d and Fig 2b). When the scaled matrix relaxation time $A=\frac{\tau_{\mathrm{a}}}{\tau_{\mathrm{m}}} \gg 1$ : if the scaled proliferation pressure $j=\frac{\tau_{\mathrm{g}}}{\tau_{\mathrm{t}}} \ll 1$, the tissue grows as a stable spheroid (bottom right of Fig. $2 \mathrm{i}$ and bottom panel of Extended Data Fig. 11b); if the scaled proliferation pressure $j=\frac{\tau_{\mathrm{g}}}{\tau_{\mathrm{t}}} \sim O(1)$, the growth is unstable and the tissue breaks symmetry and develops branches (bottom panel of Fig.1d and bottom panel of Fig. $2 \mathrm{~b}$ and $3 \mathrm{~b}$ ); if the scaled proliferation pressure $j=\frac{\tau_{\mathrm{g}}}{\tau_{\mathrm{t}}} \gg 1$, the morphological stability of the tissue depends on $\mu=\frac{\mu_{\mathrm{t}}}{\mu_{\mathrm{m}}}$ (see Extended Data Fig11d,e and 13c); for $\mu=\frac{\mu_{\mathrm{t}}}{\mu_{\mathrm{m}}} \ll 1$, the tissue remains spheroidal (Extended Data Fig.11d,e, 13c); for $\mu=\frac{\mu_{t}}{\mu_{m}} \gg 1$, growth is unstable and the tissue breaks symmetry and develops branches (Extended Data Fig.11d,e, 13c). We have shown representative images from the experiments and the simulations in different regimes of the Phase diagram; one set of images from stable tissues in the blue region 
$547\left(A=\frac{\tau_{\mathrm{a}}}{\tau_{\mathrm{m}}}=0.4, \mu=\frac{\mu_{\mathrm{t}}}{\mu_{\mathrm{m}}}=0.002, j=\frac{\tau_{\mathrm{g}}}{\tau_{\mathrm{t}}}=0.05\right)$; top left is first set of unstable images from a specific 548 point $\left(A=\frac{\tau_{\mathrm{a}}}{\tau_{\mathrm{m}}}=400, \mu=\frac{\mu_{\mathrm{t}}}{\mu_{\mathrm{m}}}=2, j=\frac{\tau_{\mathrm{g}}}{\tau_{\mathrm{t}}}=0.22\right)$; and top right is second set of images of another 549 unstable point $\left(A=\frac{\tau_{\mathrm{a}}}{\tau_{\mathrm{m}}}=3.3, \mu=\frac{\mu_{\mathrm{t}}}{\mu_{\mathrm{m}}}=2, j=\frac{\tau_{\mathrm{g}}}{\tau_{\mathrm{t}}}=0.14\right)$. Scale bars are $200 \mu \mathrm{m} . \mathrm{p}<0.05^{*} ; \mathrm{p}<0.01$ $550^{* *} ; \mathrm{p}<0.001^{* * *}$. 
bioRxiv preprint doi: https://doi.org/10.1101/2022.01.19.476771; this version posted January 20, 2022. The copyright holder for this preprint (which was not certified by peer review) is the author/funder, who has granted bioRxiv a license to display the preprint in perpetuity. It is made available under aCC-BY-NC-ND 4.0 International license.

a
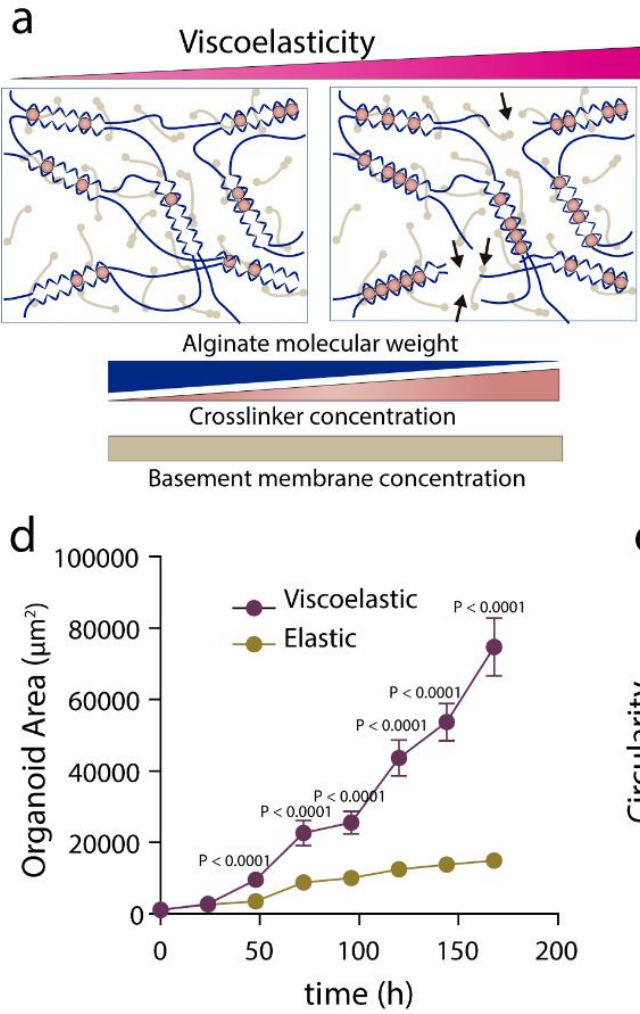

g
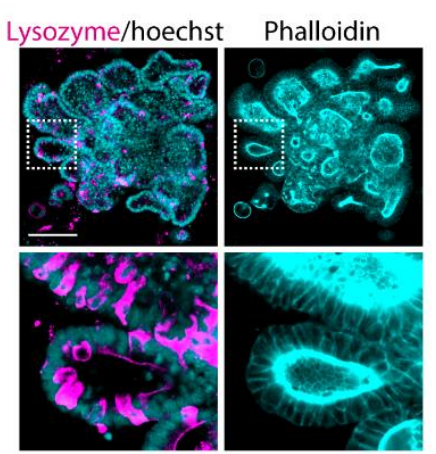

j

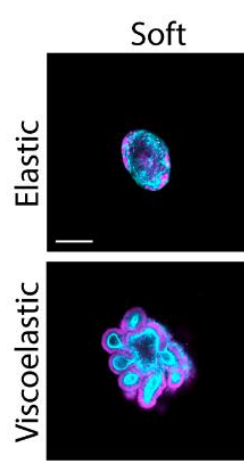

h

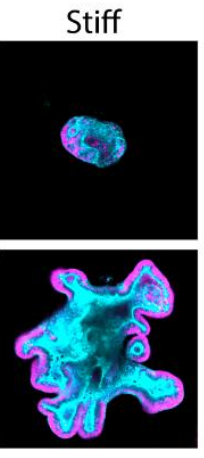

$\mathrm{k}$

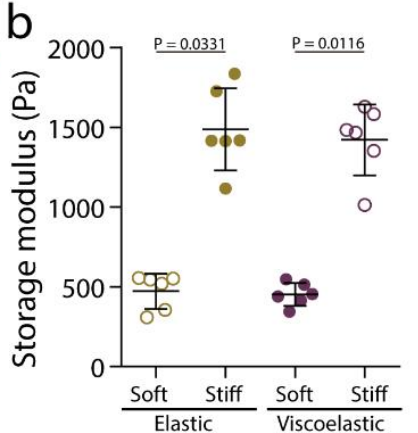

$e_{1.5}$

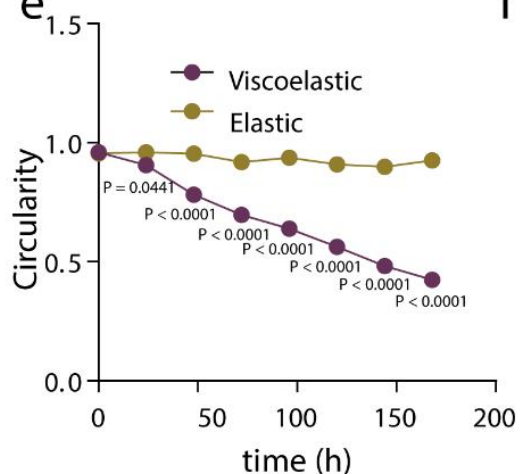

C

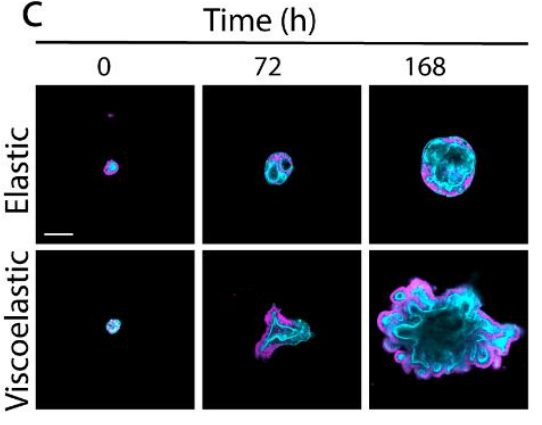

$f$

Lgr5+/Hoechst Phalloidin
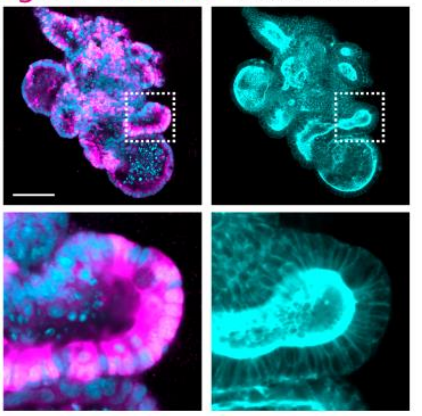
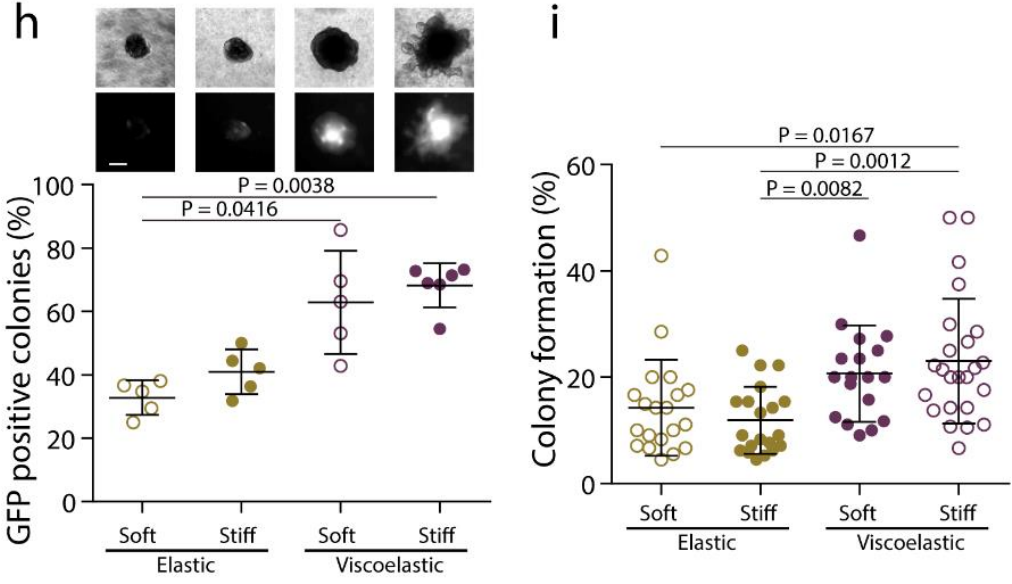

552
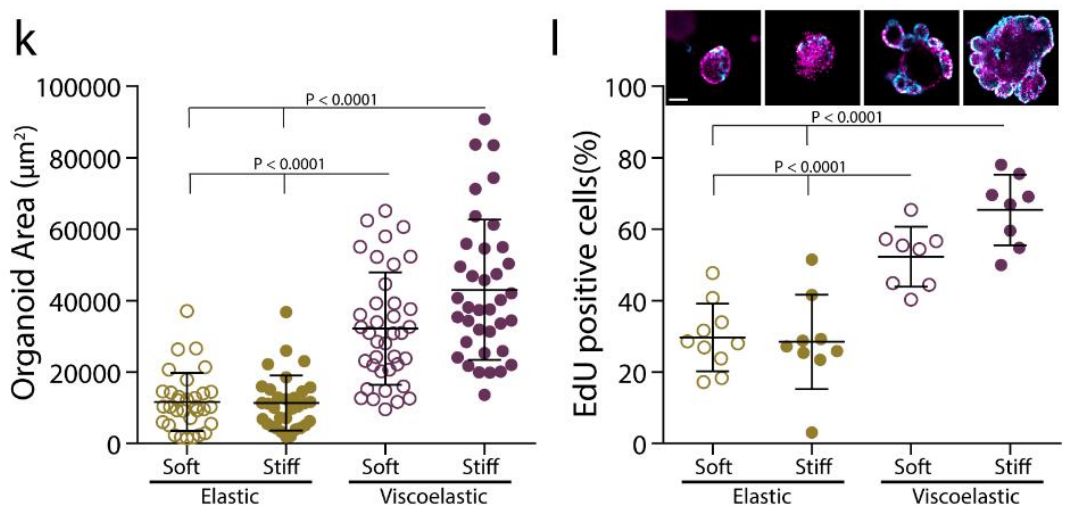
553 Figure 4: Matrix viscoelasticity controls intestinal organoid growth, symmetry breaking, 554 budding and cell patterning.

555 a, Schematic depicting of interpenetrating networks (IPNs) of alginate and Matrigel used in 556 organoid studies. Viscoelasticity is controlled by polymer molecular weight and crosslinker 557 concentration, while the concentration of Matrigel is maintained constant. $\mathbf{b}$, Storage moduli of 558 the elastic and viscoelastic alginate-matrigel IPNs. $n=6$ gels per condition. Statistical analysis 559 was performed using Mann-Whitney U-test. c, Representative examples of phalloidin and 560 hoechst stainings of intestinal organoids in elastic and viscoelastic hydrogels over 7 days of 561 culture. Phalloidin in cyan, Hoechst in magenta. d-e, Quantification of the organoids area (d) 562 and circularity (e), respectively, over 7 days in elastic and viscoelastic matrices (error bars, 563 s.e.m). $n=24 / 26,2 / 24,27 / 22,31 / 21,19 / 23,22 / 29,21 / 26$ organoids in Elastic/Viscoelastic gels per 564 day. Statistical analysis was performed using Kruskal-Wallis test followed by post hoc Dunn's 565 test. f, Example of Lgr5+, phalloidin and hoechst staining of intestinal organoids in a stiff 566 viscoelastic gel after 7 days. Left, Lgr5+ (magenta) and hoechst (cyan); right, phalloidin (cyan).

567 g, Example of Lysozyme, phalloidin and hoechst staining of intestinal organoids in a stiff 568 viscoelastic gel after 7 days. Left, lysozyme (magenta) and hoechst (cyan); right, phalloidin 569 (cyan). h, Representative examples of phase contrast and Lgr5+ GFP images (upper row) and 570 quantification of GFP positive Lgr5+ intestinal organoids in the viscoelastic and elastic matrices 571 of different stiffness. $n=5,5,5,6$ samples per condition. Statistical analysis was performed using 572 Kruskal-Wallis test followed by post hoc Dunn's test. i, Quantification of the percentage of 573 colony formation per condition. $n=20,20,18,24$ images per condition. Statistical analysis was 574 performed using Kruskal-Wallis test followed by post hoc Dunn's test. j, Examples of phalloidin and Hoechst stainings of intestinal organoids in different stiffness elastic and viscoelastic matrices after 7 days. k, Quantification of the organoids area in different stiffness elastic and viscoelastic matrices. $n=32,32,38,37$ organoids per condition. Statistical analysis was performed using Kruskal-Wallis test followed by post hoc Dunn's test. $\mathbf{m}$, Example of EdU (cyan) and Hoechst (nuclei) (upper row) and the percentage of EdU positive cells (lower row) of intestinal organoids in different stiffness elastic and viscoelastic matrices. $n=10,9,8,8$ organoids per condition. Statistical analysis was performed using Kruskal-Wallis test followed by post hoc Dunn's test. All data are mean \pm s.d. except where indicated, all scale bars are $100 \mu \mathrm{m}$. 